FERMILAB-TM-2042

\title{
On Transverse Mode Coupling Instability in VLHC
}

\author{
V.V. Danilov and V.D. Shiltsev \\ Fermi National Accelerator Laboratory \\ P.O. Box 500, Batavia, Illinois 60510
}

January 1998 


\section{Disclaimer}

This report was prepared as an account of work sponsored by an agency of the United States Government. Neither the United States Government nor any agency thereof, nor any of their employees, makes any warranty, expressed or implied, or assumes any legal liability or responsibility for the accuracy, completeness, or usefulness of any information, apparatus, product, or process disclosed, or represents that its use would not infringe privately owned rights. Reference herein to any specific commercial product, process, or service by trade name, trademark, manufacturer, or otherwise, does not necessarily constitute or imply its endorsement, recommendation, or favoring by the United States Government or any agency thereof. The views and opinions of authors expressed herein do not necessarily state or reflect those of the United States Government or any agency thereof.

\section{Distribution}

Approved for public release; further dissemination unlimited. 


\title{
On Transverse Mode Coupling Instability in VLHC
}

\author{
V.V. Danilov, V.D. Shiltsev \\ Fermi National Accelerator Laboratory \\ P.O. Box 500, Batavia, Illinois 60510 \\ February 6, 1998
}

\begin{abstract}
We present the results of calculation of transverse mode coupling instability (TMCI) thresholds for Very Large Hadron Collider (VLHC). The estimates are done with use of conventional approach and more sophisticated mode dynamics analysis. We found the RF system parameters which allows to get appropriate TMCI threshold number of particles per bunch and attain high luminosity of the collider. Alternative ways to raise the threshold are briefly discussed.
\end{abstract}

\section{Introduction}

The Very Large Hadron Collider(VLHC) [1] will have large circumference, that leads to domination of transverse instabilities in beam dynamics problems [2, 3]. Consideration done by J.Rogers [2] concludes that dominant single bunch effect is the transverse mode coupling instability(TMCI) driven by a broadband impedance. We redo estimates of the impedance budget and the TMCI threshold using conventional approach and discuss observations at the Tevatron in Section 2. As most of the impedance comes from resistive wall fields which are not constant over longitudinal coordinate in a bunch $s$, then more sophisticated analysis is done in Section 3 with means of the method developed in [5]. We study dependence of the beam eigenfrequencies versus current and modes coupling in the VLHC low-field option. In Section 4 we consider possible ways to increase the threshold in the VLHC.

\section{Rough estimates}

Basically, the TMCI (or "strong head-tail instability") appears due to defocusing effect of the wake fields induced by the head of the bunch on the bunch tail particles. In the presence of synchrotron motion, there is a permanent exchange of head and tail particles that helps to avoid the instability. The instability was observed at many electron storage rings which usually tend to increase the synchrotron tune $\nu_{s}$ in order to stabilize the TMCI. To date there is no solid evidence of the "stronghead tail" instability in proton machines [4]. Nonetheless, it is expected the proton TMCI will be important at injection into the SPS, when it works with LHC parameters and in the VLHC. We have 
to note that for existing proton accelerators, stabilizing processes other than the synchrotron mixing can play important role, for example, incoherent tune spread due to direct space charge and/or broad-band impedance, or other sources of possible Landau damping. They are not yet analyzed properly, and, therefore, it is not clear will they distort the picture of the TMCI at the VLHC or not.

After these precautions, we start with routine analysis of the mode coupling phenomena.

The transverse mode-coupling instability leads to fast beam break-up, and, therefore, sets strong constraint on a broad band transverse impedance $Z_{\perp}$. The threshold number of particles per bunch $N_{p}$ can be estimated as [6]:

$$
N_{p}^{t h}=\frac{32 \pi^{3 / 2}(E / e) \sigma_{s} \nu_{s}}{3 e c \operatorname{Im}<Z_{\perp} \beta_{\perp}>}
$$

where brackets $<\ldots .>$ mean averaging with the beta-function at locations of the impedance sources.

As it was pointed out in [2], transverse broad band impedance in the low-field VLHC is dominated by resistive wall impedance. The latter can be estimated for round vacuum chamber with radius $a$ as:

$$
Z_{\perp}(\omega)=(1-i) \frac{Z_{0} \delta R}{a^{3}}
$$

where $R$ is the radius of the machine, $Z_{0}=377 \Omega$ is the vacuum impedance, $\delta=c / \sqrt{2 \pi \sigma \omega}$ is the skin depth at frequency $\omega, \sigma$ is the wall conductivity.

As the impedance depends on frequency, its effective value integrated over the bunch spectrum is used ${ }^{1}$ :

$$
Z_{\perp}=\frac{\sum_{n} Z_{\perp}\left(\omega=n \omega_{0}\right) e^{-\left(n \omega_{0} \sigma_{s} / c\right)^{2}}}{\sum_{n} e^{-\left(n \omega_{0} \sigma_{s} / c\right)^{2}}} .
$$

where $\omega_{0}=c / R$ and $\sigma_{s}$ is the rms bunch length. Using $(2,3)$ one finds that

$$
Z_{\perp}^{R W}=\frac{\Gamma(1 / 4)}{\sqrt{\pi}} Z_{\perp}\left(\omega=c / \sigma_{s}\right)=2.05 Z_{\perp}\left(\omega=c / \sigma_{s}\right) .
$$

For $\sigma_{s}=10 \mathrm{~cm}$ long bunches in $\mathrm{Al}$ vacuum chamber with conductivity of $\sigma=3.2 \cdot 10^{17} \mathrm{c}^{-1}$ the skin depth at $\omega=c / \sigma_{s}=3 \mathrm{GHz}$ is about $3.86 \mu \mathrm{m}$.

For other than round vacuum chambers, a geometry factor can be introduced. For example, it is equal to $R_{G} \approx 0.84$ for elliptical vacuum chamber with minor half-aperture $a$ two times less than the major one [7], i.e.

$$
\operatorname{Im} Z_{\perp}^{e f f} \approx 2.05 \cdot 0.84 \frac{Z_{0} R \delta\left(\omega=c / \sigma_{s}\right)}{a^{3}}=1.72 \frac{Z_{0} R \delta\left(\omega=c / \sigma_{s}\right)}{a^{3}} .
$$

Additional wide-band impedance of the ring can be estimated as a sum of impedances of its components, such as bellows, BPMs, kickers, vacuum ports, tapers, etc. Most of these elements are discontinuities having resonant frequencies much higher than the frequencies within the bunch spectrum, and, therefore, they give rise to a mostly inductive impedance. At this stage of the VLHC ring design we restrict ourselves with rough estimate, based on assumption that for every half FODO cell

\footnotetext{
${ }^{1}$ as will be discussed in the next section, this is the point of some uncertainty in the impedance estimates
} 
length of $L=250 \mathrm{~m}$, there are some discontinuities like aperture increase $\Delta=0.5 \mathrm{~mm}$ and total length of about $g=10 \mathrm{~cm}$, then

$$
\operatorname{Im} Z_{\perp}=\left(\frac{Z}{n}\right) \cdot \frac{2 R}{a^{2}}=-\frac{2 R}{a^{2}} \cdot \frac{Z_{0} g \Delta}{2 \pi R a} \times N_{\text {elements }}, N_{\text {elements }}=2 \pi R / L .
$$

Resulting impedance budgets for low-field and high-field options of the VLHC (some parameters are taken from [2]), $3 \mathrm{TeV}$ low-field injector with $C=34 \mathrm{~km}$ (with $L \simeq 86 \mathrm{~m}$ ) and for the LHC [8] are presented in Table 1.

Table 1 also presents the threshold bunch population for these machines at their injection energies where the TMCI is expected to be more dangerous.

For the low-field option of the VLHC the bunch intensity threshold determined by the TMCI (mostly due to resistive walls) is equal to:

$$
N_{t h} \approx 3.87 \cdot 10^{10} \cdot \sqrt{\frac{\sigma_{s}}{10 \mathrm{~cm}}} \cdot \frac{E}{3 \mathrm{TeV}} \cdot \frac{\nu_{s}}{0.01} \cdot\left(\frac{a}{0.9 \mathrm{~cm}}\right)^{3} \cdot \frac{550 \mathrm{~km}}{C} \cdot \frac{320 \mathrm{~m}}{<\beta>} .
$$

For example, one can expect that due to 4 times smaller beta-function and seven times longer bunch length, the threshold in $3 \mathrm{TeV}$ ring built with the same low-field magnet technology will be about 10 times the threshold of the $50 \mathrm{TeV}$ collider ring as long as acceleration ratio (top energy divided by injection energy) and $\nu_{s}$ are the same for both rings.

It is useful to apply the above consideration to the Tevatron ring (see also Table 1). Assuming stainless steel round vacuum chamber with $a=3.5 \mathrm{~cm}$, resistivity of $\rho=7.4 \cdot 10^{-7} \Omega \mathrm{m}$, the rms bunch length of about $0.5 \mathrm{~m}$ and synchrotron tune 0.002 at the injection energy of $150 \mathrm{GeV}$, $<\beta>\approx 50 \mathrm{~m}$, one gets

$$
N_{t h}^{T e V} \approx 4.6 \cdot 10^{12}
$$

that corresponds to effective resistive wall broadband impedance of about $0.8 \mathrm{M} \Omega / \mathrm{m}$. Detailed estimate of the impedance made by K.Y.Ng [9] gives total transverse impedance about 3-4 times larger. There are some experimental evidences of the longitudinal broadband impedance as large as $(Z / n)^{\mathrm{TeV}} \sim 10 \Omega$ [10]. From that one can estimate the transverse broadband impedance as $Z_{\perp}=$ $2 R(Z / n) / a^{2}=10 \mathrm{M} \Omega / \mathrm{m}^{2}$. For the given spread of the impedance of (3-10) $\mathrm{M} \Omega / \mathrm{m}$ we get the threshold bunch populations of $N_{t h}^{T e V}=(3.7-12) \cdot 10^{11}$. Particle production at the Tevatron limits maximum bunch population at the Tevatron at the level of about $3 \cdot 10^{11}$, and that probably explains why the TMCI was not observed at the Tevatron. As we mentioned at the beginning of this Section, there is other factor which may play role and increase the TMCI threshold - the spread of betatron tunes within the bunch. Such a spread takes place along the bunch (due to direct space-charge or/and broad-band impedance) as well as across the bunch (due to direct space charge, octupole magnets, beam-beam interaction, etc.) It is not clear now which of the spreads may lead to more effective stabilization. Recent numerical studies [11] of the TMCI with direct space charge tune shift $\delta Q_{s c}$ have shown that the threshold grows approximately as $\mathcal{R}=1+\Delta Q_{s c} / \nu_{s}$. For the Tevatron at injection

$$
\Delta Q_{s c}=\frac{N_{p} r_{p} R}{2 \sqrt{2 \pi} \sigma_{s} \epsilon_{N} \gamma^{2}} \approx 0.001,
$$

\footnotetext{
${ }^{2}$ in the estimate we used $a=4.5 \mathrm{~cm}$, i.e. somewhat larger than for the vacuum chamber, because the aperture is larger in the Tevatron injection and abort kickers which are thought to contribute significantly into the broad-band impedance.
} 
(here $\epsilon_{N} \approx 4 \cdot 10^{-6} \mathrm{~m}$ is the rms normalized emittance, $r_{p}=1.53 \cdot 10^{-18} \mathrm{~m}$ is the proton classical radius, and we took $N_{p}=3 \cdot 10^{11}$ ) that may give additional $50 \%$ of the threshold increase. The direct space charge tune shift and tune spread are negligible at high energies (e.g. for all machines we considered above except $3 \mathrm{TeV}$ low-field injector ring with $150 \mathrm{GeV}$ injection energy - the protons will come from the Fermilab Main Injector). It would be of interest to observe the TMCI at the Tevatron, for example, by increasing the transverse broad-band impedance of the ring and to study the tune shift and the coupling of the transverse modes.

Table 1: Broadband Impedance Model and Threshold Bunch Intensities (L-F is for low-field VLHC option, H-F - high-field option, 3TI - 3 TeV low-field VLHC injector ring, TEV is for the Tevatron)

\begin{tabular}{|c|c|c|c|c|c|c|}
\hline Parameter & & L-F & H-F & 3TI & LHC & TEV \\
\hline Circumference & $C, \mathbf{k m}$ & 554 & 102 & 34 & 26.7 & 6.3 \\
\hline Top beam energy & $E, \mathbf{T e V}$ & 50 & 50 & 3 & 7 & 1 \\
\hline Injection energy & $E_{i}, \mathbf{T e V}$ & 3 & 3 & 0.15 & 0.45 & 0.15 \\
\hline Bunch length (inj.) & $\sigma_{s}, \mathbf{m}$ & 0.06 & 0.029 & 0.43 & 0.13 & $\sim 0.5$ \\
\hline Long. emittance (inj.) & $A_{r m s}, \mathbf{e V} \cdot \mathbf{s}$ & 0.3 & 0.04 & 0.2 & 0.13 & $\sim 0.5$ \\
\hline Synchr. tune & $\nu_{s}$ & 0.01 & 0.01 & 0.01 & 0.005 & 0.002 \\
\hline Beam pipe half-size & $a, \mathbf{c m}$ & 0.9 & $1.9^{*}$ & 0.9 & $1.9^{*}$ & 3.5 \\
\hline$<\beta>$ & $\mathbf{m}$ & 320 & 255 & 82 & 71 & 50 \\
\hline Resistive wall (inj.) & $\operatorname{Im} Z_{\perp}, \mathbf{M} \Omega / \mathbf{m}$ & 235 & 0.7 & 38.5 & 0.35 & 0.8 \\
\hline Bellows, BPMS, etc. & $\operatorname{Im} Z_{\perp}, \mathbf{M} \Omega / \mathbf{m}$ & 18 & $7.3^{* *}$ & 1.2 & 5.65 & 2-9 \\
\hline Total transverse & $\operatorname{Im} Z_{\perp}, \mathbf{M} \Omega / \mathbf{m}$ & 253 & 8.0 & 39.7 & 6.0 & $3-10$ \\
\hline Total longitudinal & $Z / n, \Omega$ & 0.14 & $\mathbf{0 . 0 3 4}^{* *}$ & 0.15 & 0.14 & $2-10$ \\
\hline TMCI threshold (inj.) & $N_{p} / 10^{11}, \mathbf{E q} \cdot(\mathbf{1})$ & 0.27 & 5.5 & 2.5 & 5.4 & 3.7-12 \\
\hline RW threshold (inj.) & $N_{p}^{R W} / 10^{11}$, Eq.(10) & 0.21 & $\mathbf{n} / \mathbf{a}$ & 1.8 & $\mathbf{n} / \mathbf{a}$ & $\mathbf{n} / \mathbf{a}$ \\
\hline
\end{tabular}

*- effective radius of square beam screen $(3.4 \mathrm{~cm}$ side length) for the resistive wall estimates. **- from [2]. 


\section{Mode coupling simulations}

As we know from preceding section, the most of the transverse impedance comes from the resistive wall fields. These fields are not constant over the bunch longitudinal coordinate $s$. E.g., the corresponding wake-function is given by [12]:

$$
W(s)=-\frac{2 C}{\pi a^{3}} \sqrt{\frac{c}{\sigma s}}
$$

One can see, that the wake field has a singularity $\propto 1 / \sqrt{s}$. That plays certain role in excitation and coupling of particular modes, and the averaging over the bunch spectrum is not completely correct: in fact, such averaging should be performed over different modes of transverse motion, which are not known a priori. We used different approach to pursue the problem, following the method developed in [5].

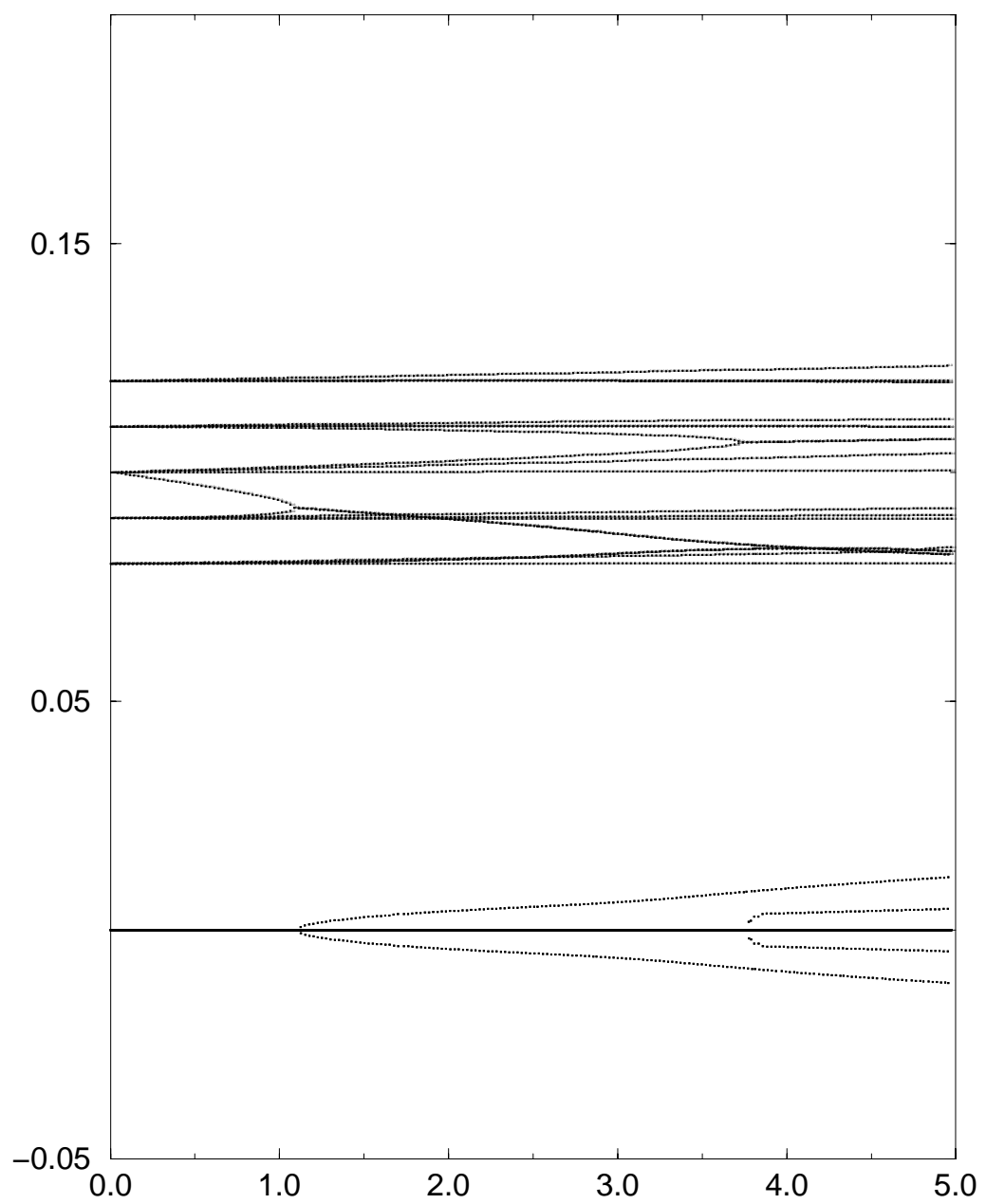

Figure 1: Eigentunes (vertical axis) versus number of protons per bunch (horizontal axis). The fractional part of the betatron tune is 0.1 ; upper lines present real part, and lower ones - imaginary part of eigentunes. 
In our simulations, the bunch is divided into 4 radial and 5 azimuthal parts, so it is possible to see the behavior of the first 4 radial and 5 azimuthal modes. It gives about few percent deviation from the exact threshold current value for the first $(0,+1,-1)$ modes coupling (see details in [5]). The integrated wake kick Eq.(9) is applied once over the revolution period, that is a good approximation while the tune shifts due to impedance and synchrotron tune are much less than 1 . The rest of the lattice is presented as a linear transform matrix. Total transformation matrices including the kick are different for different modes. Eigenvalues (eigentunes) of these modes can be calculated numerically.

In Fig.(1) one can see the eigentunes versus number of protons per bunch. The parameters used in this simulation are $\left\langle\beta>=600 \mathrm{~m}, \nu_{s}=0.01, \sigma_{s}=0.1 \mathrm{~m}, E=3 \mathrm{TeV}, C=550 \mathrm{~km}\right.$. The fractional part of the betatron tune is equal to $\nu_{\beta}=0.1$. We found that being far from low-order resonances, the tune is not significant for the threshold estimations. There is a number of "radial" eigentunes with zero "azimuthal" number around the betatron frequency at $\nu_{\beta} \pm \nu_{s}, \pm 2 \nu_{s}$. All these modes for small current have zero oscillations of the dipole moment over the angle in synchrotron phase space, and they have different dependence on the dipole moment over the synchrotron amplitude ("radius" in synchrotron phase space). For each synchrotron sideband, there are several higher "azimuthal" modes, whose tunes for small current are different from the betatron tune on a particular integer number of synchrotron tunes. This number means the eigenmode variation numbers over the angle in the synchrotron phase space. As in the case of "zero" azimuthal modes, there are a lot of "radial" modes for every azimuthal number.

With chosen parameters, the first merging of some "zero" radial mode and some "-1" radial mode occurs for the number of protons in bunch equal to $1.2 \cdot 10^{10}$. The next merging occurs for approximately triple current ("0" radial and " +1 " radial modes). In both cases a pair of the modes with equal real parts of the tune and with opposite imaginary parts of the tune appears; this, evidently, gives unstable motion of the bunch.

Let us take into account the reduction factor of $R_{G}=0.84$ due to the beam pipe ellipticity, and present the threshold obtained in the same form as Eq.(7):

$$
N_{t h} \approx 2.7 \cdot 10^{10} \cdot \sqrt{\frac{\sigma_{s}}{.1 \mathrm{~m}}} \cdot \frac{E}{3 \mathrm{TeV}} \cdot \frac{\nu_{s}}{0.01} \cdot\left(\frac{a}{0.9 \mathrm{~cm}}\right)^{3} \cdot \frac{550 \mathrm{~km}}{C} \cdot \frac{320 \mathrm{~m}}{<\beta>},
$$

This value is about 0.7 times the value we got with straightforward estimates above. Again, worrisome intensity limitation in the VLHC occurs at the injection energy.

\section{Luminosity and safety factor.}

Now we can estimate luminosity of the low-field VLHC under assumption that the number of particles per bunch $N_{p}$ remains the same during the acceleration, normalized transverse emittance $\epsilon_{n}=$ $2.5 \mathrm{~mm} \cdot \mathrm{mrad}$ (or $95 \%$ emittance of $15 \pi \mathrm{mm} \cdot \mathrm{mrad}$ ), beta-function at the interaction point of $\beta^{*}=$ $10 \mathrm{~cm}$, revolution frequency $f_{0}=0.5 \mathrm{kHz}$, and the collision energy of $50 \mathrm{TeV}\left(\gamma_{p}=5.1 \cdot 10^{4}\right)$ :

$$
\mathcal{L}=f_{0} N_{b} \frac{N_{p}^{2} \gamma_{p}}{4 \pi \epsilon_{n} \beta^{*}} \mathcal{F}\left(\frac{\sigma_{s}}{\beta^{*}}\right),
$$

where the geometrical luminosity reduction factor for round beams is $\mathcal{F}(x)=\mathcal{L}\left(\sigma_{s}\right) / \mathcal{L}(0)=$ 
$\sqrt{\pi} x e^{x^{2}} \operatorname{erfc}(x), x \equiv \beta^{*} / \sigma_{s}$. For $7 \mathrm{~cm}$ rms bunch length it leads to $\mathcal{F} \approx 0.82$ and one gets the luminosity of

$$
\mathcal{L} / 10^{34}\left[s^{-1} \mathrm{~cm}^{-2}\right]=0.73 \cdot \frac{N_{b}}{10^{5}} \cdot\left(\frac{N_{p}}{10^{10}}\right)^{2} .
$$

Number of interactions per crossing due to inelastic interaction of protons with $132 \mathrm{mb}$ cross section is about

$$
n_{\text {int }}=28 \cdot\left(\mathcal{L} / 10^{34}\right) \cdot \frac{10^{5}}{N_{b}} .
$$

One can see, from this formula, that, for the same luminosity,larger number of bunches is preferable in order to decrease the number of particles per bunch and $n_{\text {int }}$. The same preference occurs for the parameter of the beam-beam interaction:

$$
\xi=\frac{r_{p} N_{p}}{4 \pi \epsilon_{n}} \approx 0.0005 \cdot \frac{N_{p}}{10^{10}}
$$

although the bunch intensity limitation due to the TMCI at the injection appears well before beambeam effects would be of importance.

Let us choose for definiteness the frequency of the RF accelerating cavities to be $f_{R F}=500$ $\mathrm{MHz}^{3}$, it is equal to $h=9 \cdot 10^{5}$ harmonics of the revolution frequency. If one fills every 9 th bucket then number of bunches is $N_{b}=10^{5}$ and bunch spacing is $18 \mathrm{~ns}$.

Other parameters of the collider are as follow: the half FODO cell length $L=250 \mathrm{~m}$, betatron phase advance per cell $\psi=90^{\circ}$, average beta-function $\langle\beta\rangle=318 \mathrm{~m}$, tune of the 554km-long machine is about $\nu \approx 275$ that yields a momentum compaction factor $\alpha \approx 1 / \nu_{x}^{2}=1.3 \cdot 10^{-5}$.

In order to have synchrotron tune at the injection (at $3 \mathrm{TeV}$ ) of

$$
\nu_{s}=\sqrt{\frac{\alpha h e V_{0}}{2 \pi E_{i n j}}}=0.01,
$$

one needs to have the total RF voltage of $V_{0}=160 \mathrm{MV}$. That value correspond to some 10 minutes ramp of the proton energy from $3 \mathrm{TeV}$ to $50 \mathrm{TeV}$. With $V_{0}=640 \mathrm{MV} \mathrm{RF}$ voltage, the ramp is four times faster and the synchrotron tune is 0.02 .

Using all parameters presented above and Equation (10), we can calculate a safety factor of $S=N_{t h} / N_{p}$ versus luminosity (see Fig.2):

$$
S=\frac{1.79}{\sqrt{\mathcal{L} / 10^{34}}} \quad \text { at } V_{0}=160 M V
$$

and

$$
S=\frac{3.01}{\sqrt{\mathcal{L} / 10^{34}}} \quad \text { at } V_{0}=640 M V
$$

For example, the safety factor of $S=2$ corresponds to $\mathcal{L}=8 \cdot 10^{33} \mathrm{~s}^{-1} \mathrm{~cm}^{-2}$ with $V_{0}=160$ $\mathrm{MV}$ and $\mathcal{L}=2.26 \cdot 10^{34} \mathrm{~s}^{-1} \mathrm{~cm}^{-2}$ with $V_{0}=640 \mathrm{MV}$ at the injection.

\footnotetext{
${ }^{3}$ powerful DC klystrons are available for such frequency at present time, both normal- and super-conducting RF technologies at this frequency are well developed for electron-positron storage rings
} 


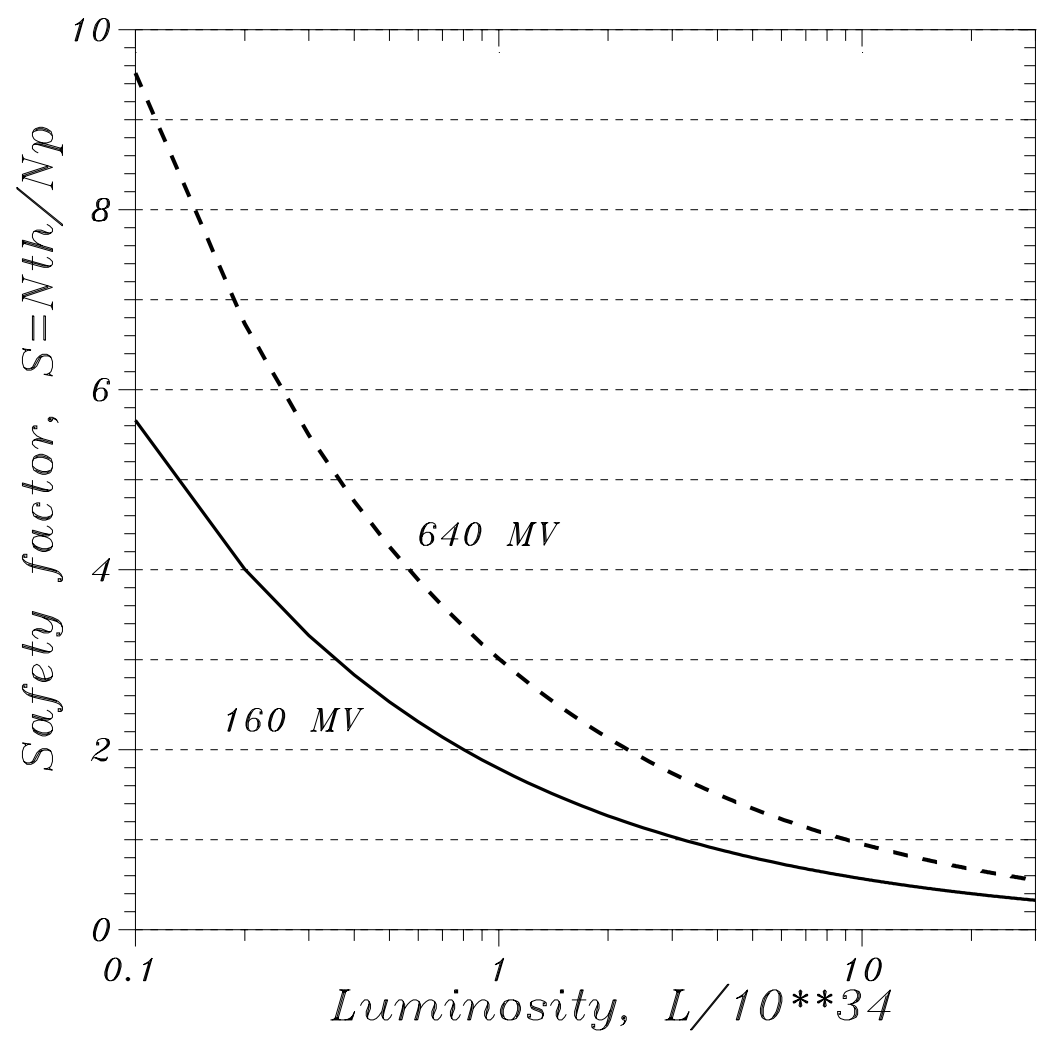

Figure 2: Safety factor $S=N_{t h} / N_{p}$ vs luminosity. RF voltage at injection is $160 \mathrm{MV}$ (solid line) and $640 \mathrm{MV}$ (dashed line).

The scaling of the safety factor $S$ for fixed luminosity is:

$$
S \propto a^{3} f_{R F}^{7 / 8} V_{0}^{3 / 8}<\beta>^{1 / 4} A^{1 / 4} .
$$

Thus, the most attractive ways are to increase the dipole magnet aperture or the RF frequency. Unfortunately, there is not too much freedom to vary these parameters, and therefore, it would be very useful to consider other ways of improvement.

\section{Ways to increase the TMCI threshold.}

There were several attempts in the past to increase the TMCI threshold with use of a feedback system. A resistive feedback doubles the threshold in PEP (see [13] and references therein) and in VEPP-4M storage rings [14]. Sophisticated reactive feedback (oscillator-like) was tested at LEP (CERN) and raised the threshold by 5\% [15]. The conventional feedback at LEP didn't help for several reasons. Due to noises (or due to some other reasons) it didn't work in essentially resistive variant, but for reactive regime of work the "-1" mode became unstable. The calculations also gave the instability of " +1 " azimuthal mode for near-threshold current.

The difference for situation with the TMCI at LEP in comparison with the other machines is its large synchrotron tune and large impedance between pickup and kicker. The VLHC parameters 
are closer to the VEPP-4M parameters, than to the LEP ones due to the fact, that their synchrotron tunes are of the same order. So, it can be assumed, that conventional feedback has to help at VLHC, as it does at the VEPP-4M collider. Special variants of feedbacks may also help to damp the TMCI [5].

One more opportunity to counteract effectively the TMCI was considered recently in Ref.[16]. Introduction of a correlated tune spread from head to tail of the bunch using RF quadrupoles has shown significant increase of the threshold if the spread is several times the synchrotron tune $\nu_{s}$. The idea is similar to the BNS-damping in linear electron-positron colliders [17] which was experimentally proven as an effective way to counteract beam break-up in SLAC Linear Collider.

\section{Acknowledgments}

We sincerely acknowledge useful comments of Gerry Jackson, John Marriner and Glenn Goderre on the Tevatron parameters and the TMCI experience at hadron machines. We thank K.Y.Ng for providing us with the Tevatron broad band impedance estimates.

\section{References}

[1] G.W.Foster, E.Malamud, "Low-Cost Hadron Colliders at Fermilab”, FNAL TM-1976 (1996).

[2] J.Rogers, "Collective Effects and Impedances in the RLHC(s)", Proc. of the DPF/DPB Summer Study on New Directions for High Energy Physics Snowmass '96.

[3] W.Chou, J.Griffin, "Impedance Scaling and Impedance Control”, FERMILAB-Conf-97-197, and Proc. 1997 Part. Accel. Conf., Vancouver (1997).

[4] see S.Peggs, "Summary of the Beam Dynamics Group", in Proc. Work. on Collective Effects in Large Hadron Colliders, CERN (1994), Particle Accelerators, vol.50 (1995), p.19.

[5] V.V.Danilov and E.A.Perevedentsev, "Feedback System for Elimination of the Transverse Mode Coupling Instability", NIM A, 391 (1997) p.77-92.

[6] see, e.g. PEP-II: An Asymmetric B-Factory, Conceptual Design Report, SLAC-418, LBLPUB-5379 (June 1993).

[7] L.Palumbo, V.Vaccaro, M.Zobov, "Wake Fields and Impedances" LNF-94/041 (1994) and CERN Accelerator School, 5th Advanced Course, CERN 95-06 (1995), p.331.

[8] F.Ruggiero, "Single Beam Collective Effects in the LHC", CERN SL/95-09 (AP), LHC Note 313 (1995).

[9] K.Y.Ng, "Beam Stability Issues of the Tevatron", Report presented at the Fermilab III Technical Advisory Committee Second Meeting (July 1995).

[10] G.Jackson, private communication (1998). 
[11] M.Blaskiewicz, "Effects of Incoherent Space Charge Forces on the Fast Head-Tail Instability", Proc. 1997 IEEE PAC, Vancouver (1997).

[12] A.W.Chao, "Physics of Collective Beam Instabilities in High Energy Accelerators", John Wiley \& Sons, Inc., 1993

[13] S.Mayers, "Stabilization of the Fast Head-Tail Instability by Feedback", Proc. 1987 IEEE PAC, Washington DC (1987), p.503.

[14] M.M.Karliner, et al.,"Feedback System for Elimination of the Fast Head-Tail Instability at Storage Ring VEPP-4M", Proc. 1996 European Particle Accelerator Conference, Barcelona (1996).

[15] L.Arnaudon, et al., "First Test with a New Transverse Reactive Feedback System", CERN SL-MD Note 158 (1994)

[16] V.V.Danilov, "On Possibility to Increase the TMCI Threshold by RF Quadrupole", FNAL TM2033 (1997).

[17] V.Balakin, A.Novokhatsky, V.Smirnov, "VLEPP: Transverse Beam Dynamics", Proc. of 12th Int. Conf. on High-Energy Accelerators, Fermilab (1983), p.119. 